\title{
A heat balanced sigma-delta uncooled bolometer
}

\author{
M Denoual ${ }^{1}$, D Brouard ${ }^{1}$, A Veith ${ }^{1}$, O De Sagazan ${ }^{2}$, M Pouliquen ${ }^{1}$, \\ P Attia ${ }^{3}$, E Lebrasseur $^{4}$, Y Mita $^{4}$ and G Allègre ${ }^{1}$ \\ ${ }^{1}$ GREYC-ENSICAEN, Université de Caen Basse Normandie, 6 bd maréchal Juin, F-14000 Caen, France \\ ${ }^{2}$ IETR Groupe Microélectronique, Université de Rennes 1, F-35000 Rennes, France \\ ${ }^{3}$ NXP-Semiconductor, Colombelles, France \\ ${ }^{4}$ University of Tokyo, Tokyo, Japan \\ E-mail: mdenoual@ensicaen.fr
}

\begin{abstract}
This paper describes a heat balanced bolometer with an integrated CMOS sigma-delta interface. The sigma-delta interface accomplishes both the analogue-to-digital conversion and the heat feedback and therefore no other digital core is required. The heat feedback is implemented using a capacitively coupled electrical substitution technique with a digitally modulated signal that enables feedback linearization. Proof of concept is experimentally demonstrated with a polymer-type resistive bolometer and infrared optical source.
\end{abstract}

Keywords: uncooled bolometer, heat balanced, sigma-delta interface, digital electrical substitution

\section{Introduction}

Uncooled resistive bolometers are one type of infrared sensor $[1,2]$. Their detection principle is based on first the conversion of the optical infrared power into heat onto a thermally insulated membrane and then on a temperature measurement via a sensing resistor to monitor the heat variation $[1,2]$.

Uncooled resistive bolometers represent $95 \%$ of the market of infrared imaging [3], which finds applications in process control, security, automotive safety and building thermal budgeting. This huge market share is mainly due to a low manufacturing cost with easy integration in semiconductor process lines. The past decades have focused on the material optimization for sensitivity improvement and on optimization of the read-out circuitry (read-out integrated circuit). This research and development work is driven by time response and power consumption concerns as well as by the willingness to turn uncooled infrared detectors into smart detectors able to compensate for process discrepancies [4] and that integrate the analogue-to-digital conversion [5, 6] or other features. In these research and development works, the uncooled resistive bolometer is used in an open-loop mode.
In order to meet expectations in terms of time response and process discrepancies compensation, a complementary approach is to use the uncooled resistive bolometer in the closed-loop mode. Such an approach was theoretically studied in the early 1990s [7] and experimentally demonstrated on bolometers in the late 1990 s by Rice $[8,9]$. The underlying principle is the electrical substitution (ES), also called electrical equivalence. The ES principle assumes that Joule produced electrical power can be used to compensate for fluctuation in the incoming optical power onto the resistive bolometer. Consequently, Joule power can be used to operate bolometers in the closed-loop mode.

The closed-loop mode operation is interesting since it leads to systems almost independent of the sensor characteristics and therefore much less sensitive to fabrication process discrepancies [10]. Furthermore, as the closed-loop system becomes independent of sensor characteristics, its performance such as time response or sensitivity might be tuned by control electronics. The basic architecture of the closed-loop operation is shown in figure 1 . It is a negative feedback system. It consists of the bolometer, conditioning electronics for amplification and filtering, a controller and a power feedback block. The bolometer and the conditioning 


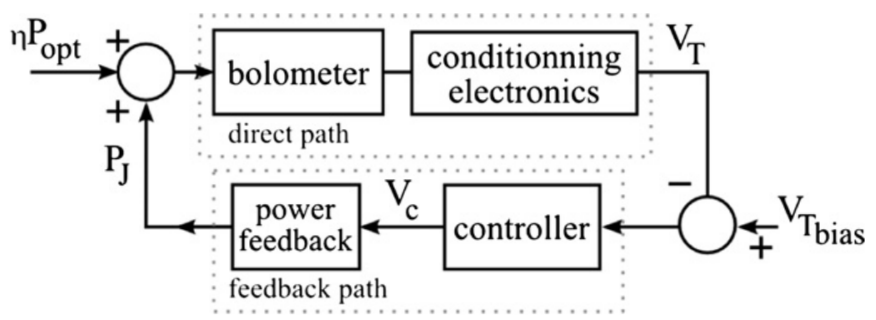

Figure 1. Architecture of a bolometer operating in the closed-loop mode.

electronics form the direct path used in the open-loop mode. The controller and the power feedback block form the feedback path. The controller is used to reduce the difference between the thermal setting point, $V_{\text {Tbias }}$, and the actual temperature of the bolometer represented by $V_{T}$. The controller output, $V_{c}$, is transformed into Joule power, $P_{J}$, and applied to the bolometer. The high gain of the controller ensures that this Joule power compensates for the incoming optical power, $\eta P_{\mathrm{opt}}$, so as to make the difference between the thermal setting point and $V_{T}$ very small. The control electronics of the controller also enables setting closed-loop performance independently of the bolometer sensor characteristics. Note that the coefficient $\eta$ represents the fraction of optical power absorbed onto the surface of the bolometer $(\eta \in[0,1])$. The denomination heat balanced bolometer refers to the force balanced one used in force feedback accelerometers [11].

Various configurations have been proposed to practically implement the electrical substitution principle in order to operate the bolometer in the closed-loop mode. In 2009, we proposed a new configuration: the capacitively coupled electrical substitution (CCES) [12]. The CCES configuration enables easier control of the closed-loop system and can be applied to existing resistive bolometers without structural change, since no extra resistor is required. Those two characteristics are advantages compared to previous configurations [13] where stability issues could arise [7, 14] or require extra resistor, i.e. extra material and process steps $[8,15]$.

First proposed with a discrete analogue implementation [12], the CCES was then demonstrated with a discrete digital implementation [16] to take advantage of the power feedback linearization induced by the digital feedback [17]. Such implementation involved microcontroller, DAC and ADC. In this paper, for the first time, we present an integrated digital implementation of the CCES with a sigma-delta interface. Such an interface enables both the digital implementation of the CCES and the analogue-to-digital conversion of the measure.

Figure 2 illustrates the feedback linearization in the case of digital implementation with a sigma-delta modulated signal as an example. It considers first in figure 2(a) a triangular shaped control voltage $V_{c}$, with an analogue implementation. The Joule power is then proportional to the square of $V_{c}\left(P_{J}\right.$ $\propto V_{c}^{2} / R_{b}$, with $R_{b}$ the resistance of the sensing resistor of the bolometer) and exhibits a parabolic shape. In the case of digital implementation (figure $2(b)$ ), the feedback voltage has a constant amplitude $\left(V_{\max }\right)$ and, in the case of sigmadelta modulation, is pulse-density modulated; it corresponds to the bitstream signal out of the sigma-delta modulator. The average value of the bitstream corresponds to $V_{c}$. The instantaneous feedback power is consequently also pulsedensity modulated with a constant amplitude $\left(G_{\mathrm{fb}}{ }^{2} V_{\max }{ }^{2} / R_{b}\right.$, with $G_{\mathrm{fb}}$ the voltage gain of the power feedback block) and its average value is linearly proportional to $V_{c}$. Therefore, the pulse-density modulated feedback signal linearizes the feedback path. The averaging of the feedback power comes from the limited thermal bandwidth of the bolometer. Indeed, the bitstream modulation frequency of the order of the $\mathrm{MHz}$ range is several orders of magnitude higher than the thermal bandwidth of bolometers (typically $20 \mathrm{~Hz}$ for thermal imaging systems). Feedback linearization leads to simpler controller design and simpler working point setting.

This paper first describes the implementation of the sigma-delta interface for the heat-balanced bolometer and its operating principle. Then results in the following section experimentally demonstrate the operating principle.

\section{Heat balanced bolometer}

\subsection{Global view of the implementation}

Figure 3 gives an overview of the heat balanced bolometer with the sigma-delta interface system. It is a mixed electrical and thermal system that operates in the closed-loop mode. It is composed of the sensor, here the bolometer where the power to voltage $(P / T / V)$ and voltage to power $(V / P)$ conversions occur. The power to voltage conversion follows the conventional principle of the bolometer with first a conversion of the absorbed optical power ( $\eta P_{\text {opt }}$ ) into heat and secondly a conversion of the temperature rise $\left(T_{b}\right)$ into voltage $\left(V_{\mathrm{Tb}}\right)$ through a sensing resistor $[1,2]$. The power to temperature conversion $(P / T)$ depends on the thermal characteristics of the bolometer as follows:

$$
\frac{T_{b}}{\eta P_{\mathrm{opt}}}=\frac{1}{G_{\mathrm{eff}}\left(1+\tau_{\mathrm{eff}} s\right)}
$$

where $G_{\text {eff }}$ and $\tau_{\text {eff }}$ are respectively the effective thermal conductance and the effective thermal constant of the bolometer.

The temperature to voltage conversion $(T / V)$ depends on the coefficient of temperature (temperature coefficient of resistance-TCR, $\alpha)$ of the sensing resistor $\left(R_{b}\right)$ and its bias current $\left(I_{B}\right)$, and is given by the following equation:

$$
\frac{V_{\mathrm{Tb}}}{T_{b}}=\alpha R_{b} I_{B}
$$

The voltage to power conversion $(V / P)$ stands for Joule heat produced by feedback into the sensing resistor according to

$$
P_{J}=\frac{V_{\mathrm{fb}}^{2}}{R_{b}} .
$$

The bolometer is the sensitive element of the chain and is followed by conditioning electronics for amplification and filtering; this association constitutes the direct path of the system.

The feedback path first includes a controller for the closed-loop performance control and adjustment. Basically, 
(a)
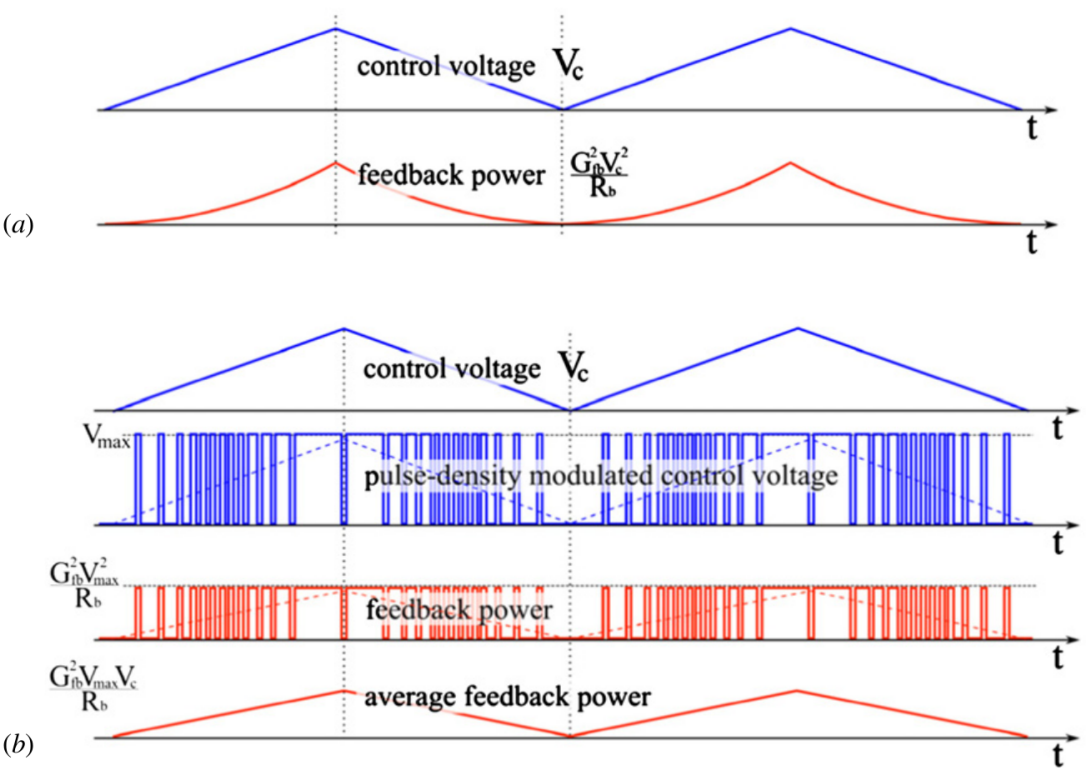

Figure 2. Chronograms illustrating the shapes of the feedback power upon analogue or digital implementation. (a) Illustration of the case of analogue implementation with $V_{c}$ being the output of the controller and the Joule feedback power in the resistor with parabolic behaviour. (b) Illustration of the case of a digital implementation. The control voltage $V_{c}$ is pulse-density modulated. The average value of the bitstream corresponds to $V_{c}$ as stressed by the dashed triangular line. The resulting Joule power on the resistor is consequently also pulse-density modulated and its average value (represented by dashed line) is linearly proportional to the control voltage $V_{c}$. $G_{\mathrm{fb}}$ is the feedback voltage gain, $R_{b}$ the resistance of the sensing resistor of the bolometer and $V_{\max }$ the maximum voltage (power supply).

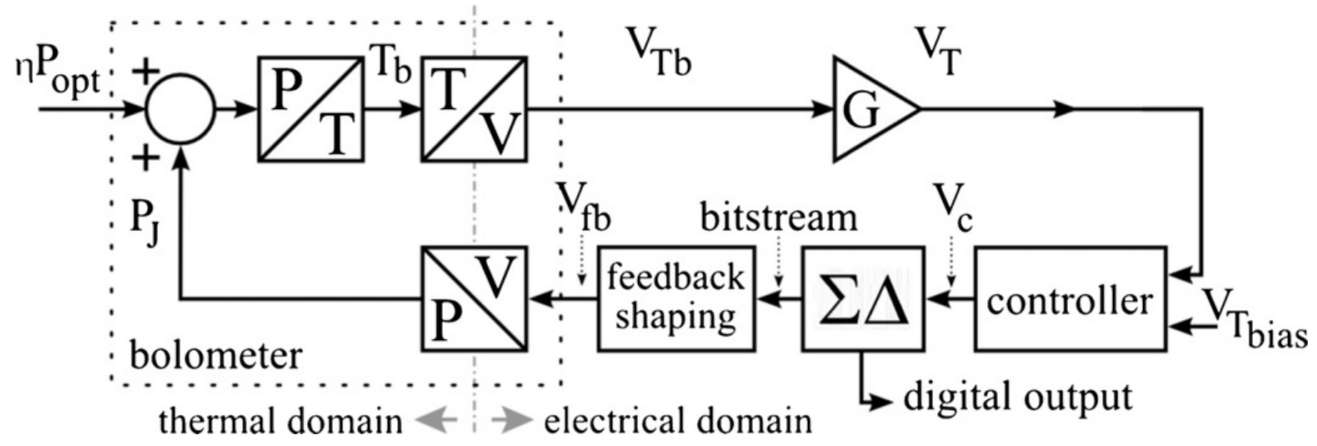

Figure 3. Block diagram of the architecture of the heat balanced bolometer with the sigma-delta interface.

the controller ensures that the difference between the bias thermal voltage, $V_{T \text { bias }}$, and the actual voltage monitoring the temperature of the bolometer, $V_{T}$, is small. Consequently, the bolometer temperature is kept constant.

The novelty in the work proposed here is the sigmadelta interface following the controller that enables both the power feedback and the analogue-to-digital conversion. The sigma-delta modulator of the interface provides a pulsedensity modulated signal, i.e. the bitstream that enables the digital implementation of the CCES without the requirement of a microcontroller or other processing unit. As illustrated in figure 2 , the pulse-density modulation linearizes the feedback path and the average Joule power filtered by the thermal bandwidth of the bolometer is linearly proportional to the controller output voltage.

The feedback shaping block enables implementation of the CCES. It shifts the bitstream signal to high frequencies by mixing it with a carrier. Doing this, the electrical and thermal working points are dissociated according to a frequency basis and both temperature sensing and power feedback can be performed on the same sensing resistor $[12,16]$.

\subsection{The sigma-delta interface}

This sub-section presents the sigma-delta integrated interface depicted in figure 4 . The detailed micro-electronics circuitry design can be found in [18]. The sigma-delta interface was realized in AMS CMOS $0.35 \mu \mathrm{m}$ technology. The chip embeds a fully differential switched-capacitor first-order sigma-delta modulator and an associated second-order cascaded integrator comb (CIC) decimator filter. These two modules perform the 8-bit analogue-to-digital conversion of the output signal.

The integrated interface also embeds circuits for the implementation of the CCES technique: a voltage controlled oscillator (VCO) for the generation of the high frequency carrier, typically over a few $\mathrm{MHz}$, and a mixer that translates the feedback signal to high frequency and a programmable attenuator. The $\mathrm{VCO}$, the mixer and the programmable attenuator compose the feedback shaping block. 


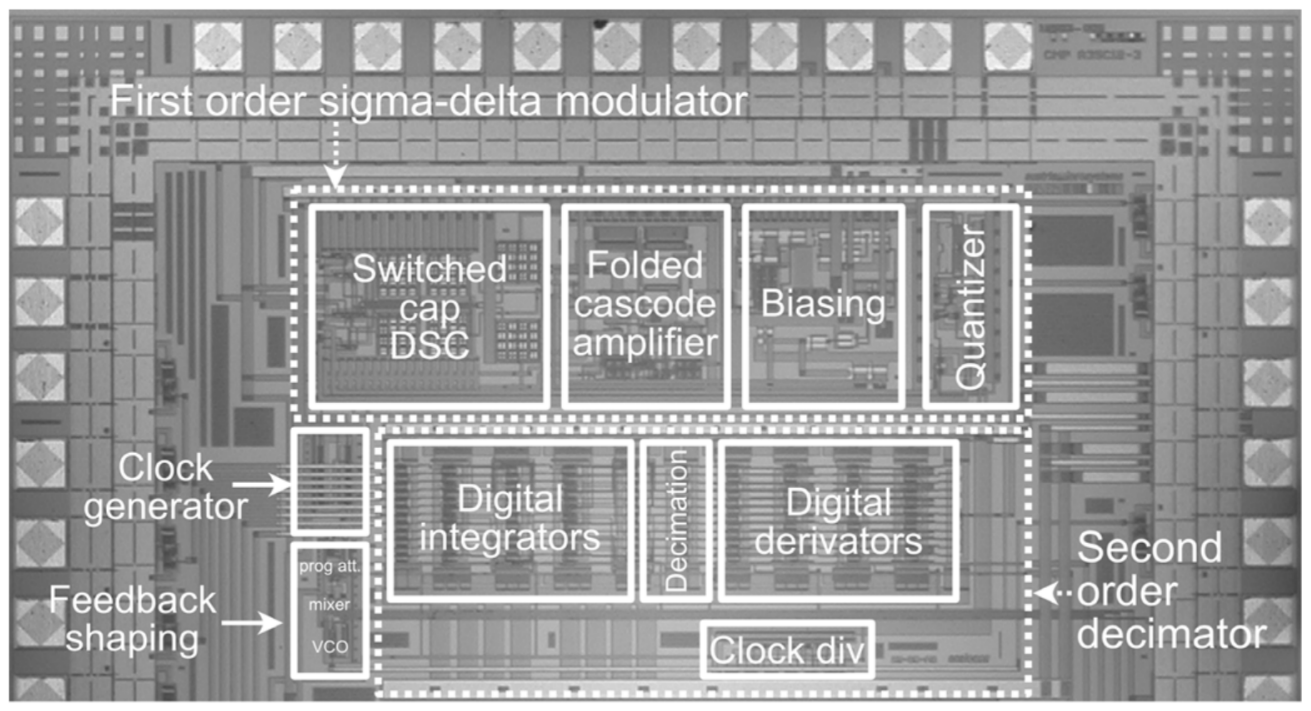

Figure 4. Microscope picture of the realized integrated sigma-delta interface for the heat balanced bolometer. The die is $2.3 \mathrm{~mm}$ wide.

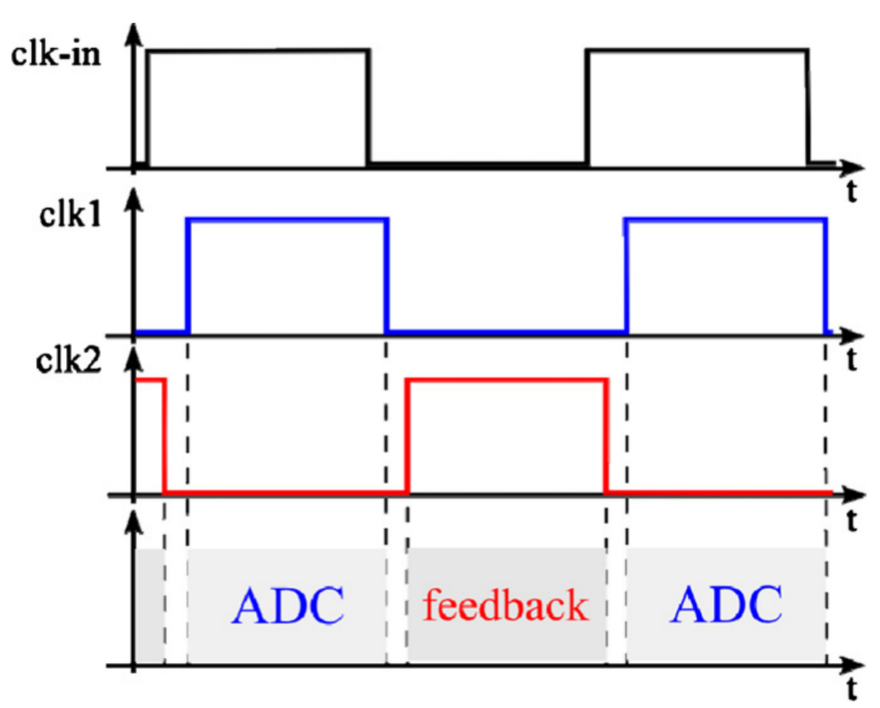

Figure 5. Chronogram of clock phases. The main clock, clk-in, produces two non-overlapping clock signals, clk1 and clk2, that individually drive two different operation steps: clk1 drives the analogue-to-digital conversion of the signal, while clk 2 drives the power feedback.

The clock generator block produces the non-overlapping clock signals, clk1 and clk2, for the switched-capacitor sigmadelta modulator. These two clock signals are also used to dissociate an input signal acquisition step from a feedback step in order to prevent any high frequency feedback into the sigma-delta modulator input during the analogue-to-digital conversion. Note that the decimation filter is clocked with clk2. Figure 5 illustrates the time multiplexing of the two-step operation, analogue-to-digital conversion and heat feedback.

\section{Experiments}

Experiments with an infrared optical source were conducted to validate the operation of an uncooled resistive bolometer with a sigma-delta interface implementing the CCES. Experiments

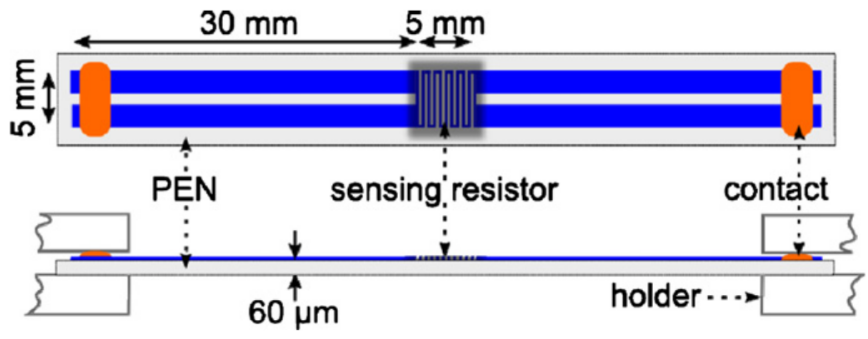

Figure 6. Top and side schematic views of the polymer membrane resistive bolometer.

were conducted at room temperature and under atmospheric pressure. Before presenting the experimental setup, this section describes the resistive bolometer used.

\subsection{Description of the uncooled bolometer}

The bolometers used for the experiments are polymer membrane-type bolometers. This choice is driven by pragmatic considerations, i.e. this kind of device is cheap, easy to fabricate and robust. The main drawback is the poor intrinsic performance in terms of thermal time constant and sensitivity. The bolometer is illustrated in figure 6. It consists of a $60 \mu \mathrm{m}$ thick, $8 \mathrm{~mm}$ wide and $7 \mathrm{~cm}$ long polyethylene naphthalate suspended ribbon. A $80 \mathrm{~nm}$ thick aluminium layer is patterned onto the surface to form the sensing resistor and the conductive paths. The sensitive resistor in the middle of the ribbon is serpentine with a $5 \mathrm{~mm} \times 5 \mathrm{~mm}$ surface. Its nominal resistance at room temperature is around $700 \Omega$ and its TCR, extracted from measurements, is $2.3 \times 10^{-3} \mathrm{~K}^{-1}$.

Dark ink is applied onto the sensing resistor in order to increase the infrared absorption coefficient $(\eta)$. The thermal characteristics of the bolometer extracted from measurements at $20 \mathrm{~m}$ Torr and room temperature are effective thermal conductance $G_{\text {eff }}=550 \mu \mathrm{W} \mathrm{K}-1$ and a thermal capacitance $C_{\text {th }}=60 \mathrm{~mJ} \mathrm{~K}^{-1}$ leading to a thermal constant $\tau_{\text {eff }}=110 \mathrm{~s}$. 


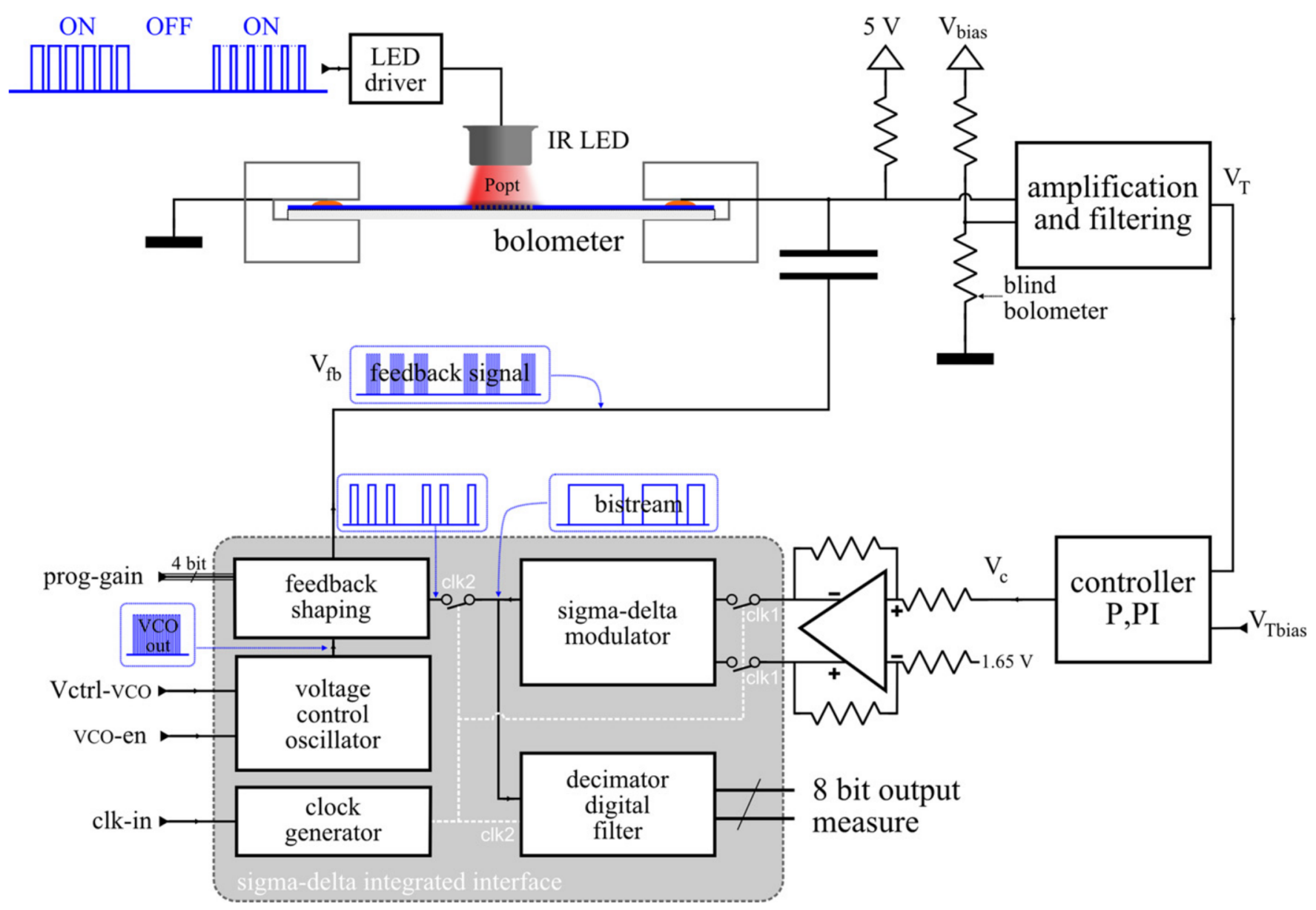

Figure 7. Schematic of the experimental setup. The typical shapes of the signals are represented in small rounded boxes connected to the signal path.

\subsection{Experimental setup}

3.2.1. Experimental setup overview. The experimental setup is depicted in figure 7. Two polymer bolometers are used. One is exposed to the infrared optical source for the measurement, the sensing bolometer, whereas another is hidden and referred to as the blind reference bolometer. The two bolometers enable a differential measurement that rejects the temperature variations of the environment. The differential signal is filtered and amplified before feeding the system controller.

The system controller consists of analogue proportional (P) or proportional-integral (PI) correctors. An interface stage allows the transition from single-ended electronics to the fully differential sigma-delta interface as well as the transition from the $5 \mathrm{~V}$ discrete circuitry to the $3.3 \mathrm{~V}$ integrated circuitry. The sigma-delta interface outputs both the feedback signal $\left(V_{\mathrm{fb}}\right)$ that is capacitively coupled to the bolometer and an 8-bit measure. The sensing bolometer is optically stimulated by an infrared LED (L7850, Hamamatsu) with $1.45 \mu \mathrm{m}$ wavelength and $1 \mathrm{~mW}$ nominal power. The optical infrared stimulus consists of a twice square modulated signal as in [16]. The low frequency modulation determines the power ON-power OFF rhythm while the duty cycle of the high frequency modulation sets the average optical power received by the bolometer. Experiments are performed at room temperature and under atmospheric pressure.
3.2.2. Experimental setup biasing and control signals. The differential bridge consisting of the sensing bolometer, the reference blind bolometer and two bias resistors is $5 \mathrm{~V}$ powered and balanced by the means of a 12-bit DAC of a NI-6009 data acquisition device. The balance of the bridge prevents the saturation of the succeeding filtering and amplification stage.

The thermal bias point is set by a voltage signal, $V_{\text {Tbias, }}$, at the input of the loop controller. This bias signal is provided by another 12-bit DAC of the NI-6009 data acquisition device. This voltage defines the working temperature of the bolometer. In practice, the working temperature is a few kelvin over room temperature, typically 1-3 K.

The sigma-delta interface is $3.3 \mathrm{~V}$ powered. The main clock for the sigma-delta modulator and the decimator filter is provided by an external signal, clk-in. When enabled by the VCO-en signal, the VCO of the sigma-delta interface oscillates between $100 \mathrm{kHz}$ and $600 \mathrm{MHz}$ depending on VctrlVCO voltage. A 4-bit input sets the programmable attenuation of the feedback shaping block.

\section{Results}

Figures $8(a)$ and $(b)$ depict typical signals at the output of the sigma-delta interface for the heat balanced bolometer. Figure $8(a)$ illustrates the time separation between feedback 


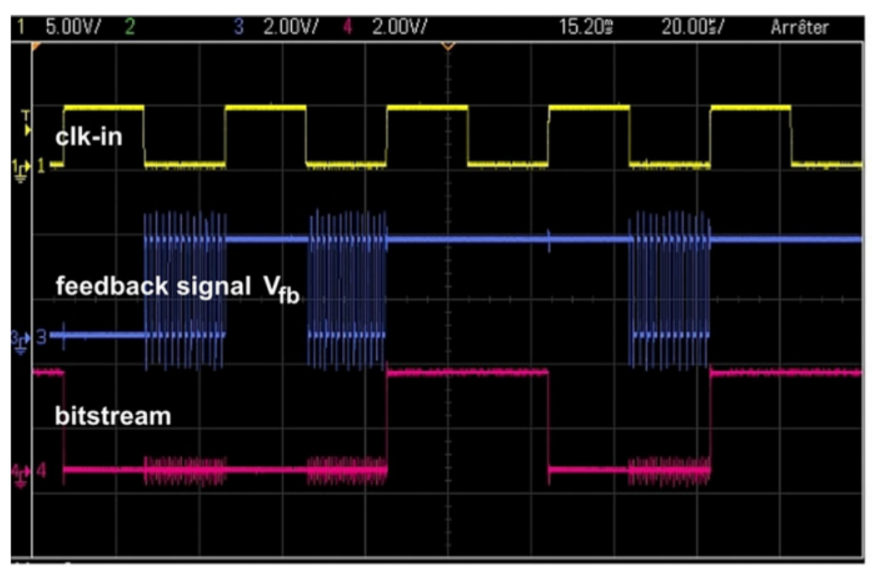

(a)

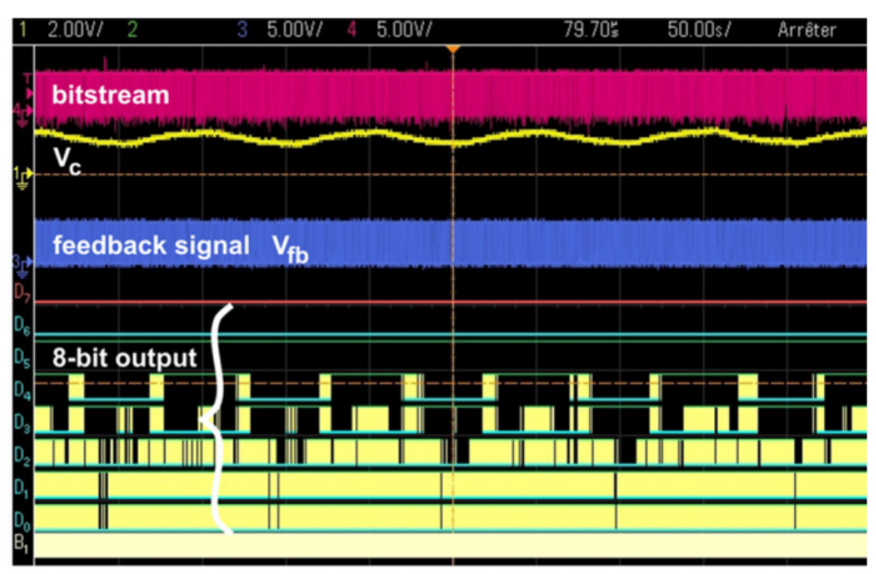

(b)

Figure 8. Oscilloscope recording of the typical signals of the sigma-delta interface for the heat balanced bolometer.

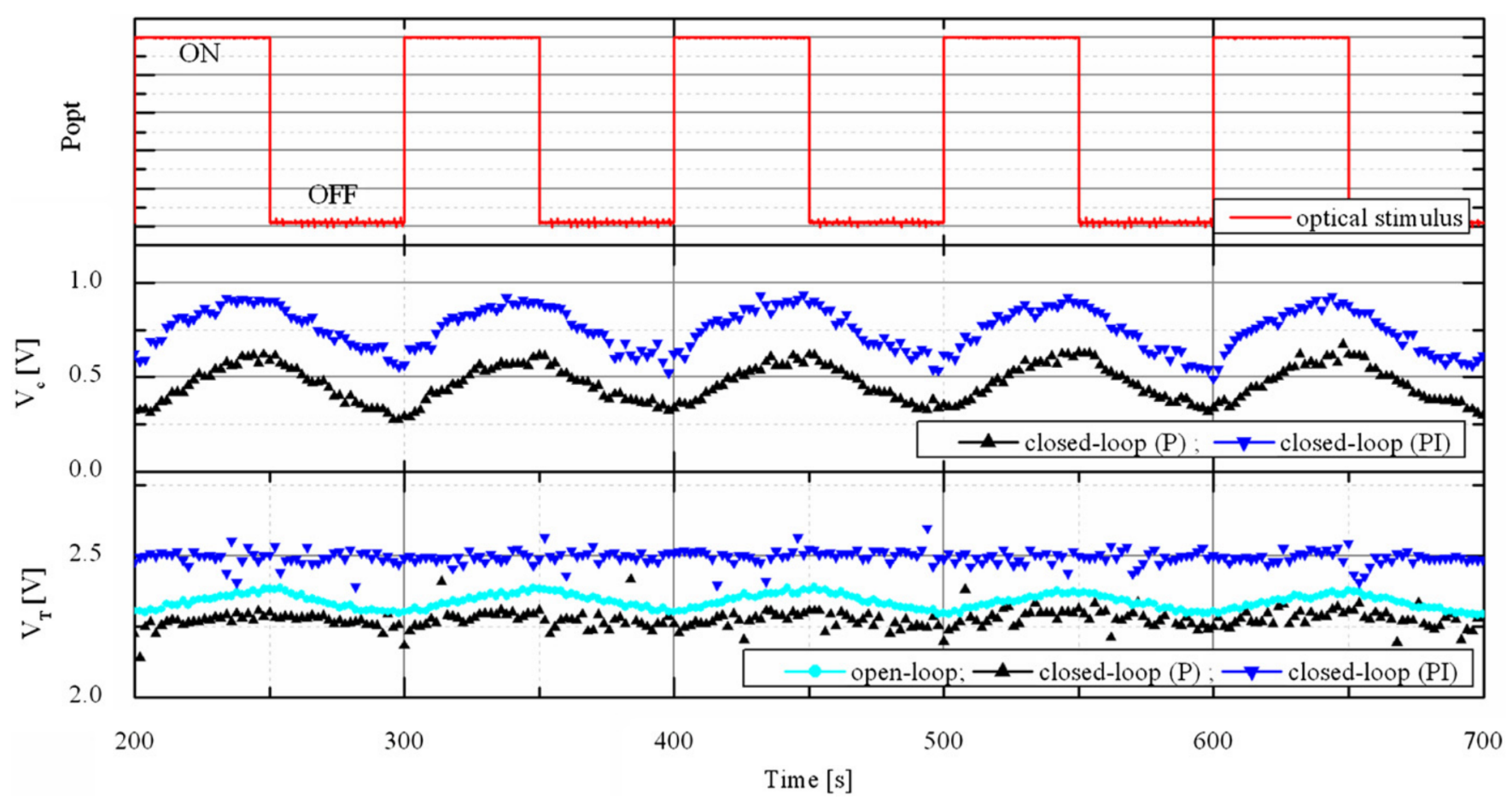

Figure 9. Response of the heat balanced bolometer to optical stimulus in open-loop and closed-loop operation modes.

and the analogue-to-digital conversion. The analogue-todigital conversion occurs during the clk1 phase corresponding to the high level of the reference clock input, clk-in. During this phase, the feedback signal is disabled. During the represented recording, the clk-in frequency was $25 \mathrm{kHz}$, and the frequency of the VCO was set around $600 \mathrm{kHz}$ with the Vctrl-VCO input. Note that the feedback is inverted relative to the bitstream, i.e. the feedback signal is active during low level of the bitstream. Indeed, the bitstream is related to temperature variations, while the feedback signal is related to the feedback power applied to the bolometer and those two quantities should vary oppositely: when the temperature rises, the feedback power should decrease to keep a constant operating temperature. Coupling can be observed between the feedback signal and the bitstream probably due to the common guard ring of the integrated chip. Nevertheless, this coupling does not affect the 8-bit output data obtained after the decimation filter.

Figure $8(b)$ represents the 8-bit digital output and the voltage at the output of the controller, $V_{\mathrm{fb}}$, thereby illustrating the dual role of the sigma-delta interface: analogue-to-digital conversion and feedback.

Figure 9 illustrates the operation of the heat balanced bolometer in two operation modes: open-loop and closed-loop. The optical infrared stimulus is the same in both cases, with an ON-OFF period of $100 \mathrm{~s}$ and optical power amplitude of $200 \mu \mathrm{W}$ defined by a $20 \%$ duty cycle of the high frequency modulation of the LED driver input signal. The frequency of the sigma-delta interface clock, clk-in, was set to $200 \mathrm{kHz}$ and 
the control voltage of the $\mathrm{VCO}$ was tuned to obtain a $2 \mathrm{MHz}$ VCO output frequency. The voltage $V_{\text {Tbias }}$ that sets the thermal working point is fixed at $2.5 \mathrm{~V}$ so as to ensure a symmetrical dynamic range. Figure 9 shows the optical stimulus signal, the voltage $V_{T}$ representing the temperature of the bolometer and the feedback signal $V_{\mathrm{fb}}$ at the output of the controller in the case of closed-loop operation.

In the open-loop mode, the temperature of the bolometer evolves following the variation of the input optical power. As expected, in the closed-loop mode the temperature variations of the bolometer are reduced when the proportional $(\mathrm{P})$ controller is used and cancelled when the PI controller is used. Furthermore, in the PI controller case the temperature is maintained at the working point defined by the setpoint $V_{\text {tbias }}$ of $2.5 \mathrm{~V}$. The time response is also reduced in closedloop mode compared to open-loop mode, here halved from $40 \mathrm{~s}$ in open-loop to $20 \mathrm{~s}$ in closed-loop (operation at atmospheric pressure explains the $40 \mathrm{~s}$ time constant compared to the $110 \mathrm{~s}$ from characterization under primary vacuum). In the closedloop operation mode, the measure is represented by the voltage at the output of the controller that is an image of the optical stimulus. This voltage is converted into an 8-bit measure output word by the sigma-delta modulator.

\section{Discussion}

The results illustrate the operating principle of a heat balanced bolometer with a sigma-delta interface, especially the dual role of the sigma-delta interface both for the analogueto-digital conversion and the feedback power generation. The experiments with simple proportional and proportionalintegral controller illustrate the typical curves obtained in closed-loop mode and validate the closed-loop operation of the complete system. The experiments demonstrate the ability to take advantage of the digital implementation without the need of a microcontroller or other processing unit; the main advantage of the digital implementation being the complete linearization of the global system. The linear property of the system enables simple design of the controller and leads to simple identification procedures in the open-loop and to a lesser extent in the closed-loop. Not only is the digital feedback ensured but also the ADC conversion.

The performance of these preliminary experiments is mainly limited by the sensor and the first version of the conditioning electronics. Still the operating principle is demonstrated and the expected performance with a future fully integrated version forecasts promising results with miniaturized bolometers, i.e. micro-bolometers, that will constitute the next step of this work.

\section{Conclusion}

In this paper, the first results of a heat balanced bolometer with an integrated sigma-delta interface are presented. Digital implementation of the capacitively coupled electrical substitution is implemented without the need of a microcontroller or any other processing unit. The experiments illustrate the expected heat balanced behaviour.
The challenge now is the use of this type of sigma-delta integrated interface with micro-bolometers exhibiting time constant of the order of $10 \mathrm{~ms}$; this is the next step of this research work.

\section{Acknowledgments}

This work was and is supported in part by the JSPS-Bridge program of the Japan Society for the Promotion of Science under no. BR-100201, the P-SOC program of the INS2I department of French CNRS under no. BFC-78518 for the sigma-delta chip fabrication and the ERE program of the French Government under no. 2012.60.090.00.470.75.01 and the CNRS DEFI instrumentation program.

\section{References}

[1] Richards P L 1994 Bolometers for infrared and millimeter waves J. Appl. Phys. 76 1-24

[2] Kruse P W 2001 Uncooled Thermal Imaging Arrays, Systems and Applications (Bellingham, WA: SPIE Optical Engineering Press)

[3] Yole Développement 2010 Uncooled IR cameras and detectors for thermography and vision Technol. Mark. Rep.

[4] Chamming's G 2009 Matrix thermal image sensor with bolometric pixel and method of reducing spatial noise $U S$ Patent Specification 20090121139

[5] Tchagaspanian M et al 2007 Design of ADC in $25 \mu \mathrm{m}$ pixels pitch dedicated for IRFPA image processing at LETI Proc. SPIE $\mathbf{6 5 4 2} 65421 \mathrm{~W}$

[6] Würfel D, Ruß M, Lerch R, Weiler D, Yang P and Vogt H 2011 An uncooled VGA-IRFPA with novel readout architecture Adv. Radiol. Sci. 9 107-10

[7] Williams C D H 1990 An appraisal of the noise performance of constant temperature bolometric detector systems Meas. Sci. Technol. 1 322-8

[8] Rice J P, Lorentz S R, Datla R U, Vale L R, Rudman D A, Sing M L C and Robbes D 1998 Active cavity absolute radiometer based on high- $T_{\mathrm{c}}$ superconductors Metrologia 35289

[9] Rice J P 2000 An electrically substituted bolometer as a transfer-standard detector Metrologia 37 433-6

[10] Denoual M and Allègre G 2010 Operating uncooled resistive bolometers in a closed-loop mode Bolometers: Theory, Types and Applications ed M W Torrence (Hauppauge, NY: Nova Science) ISBN: 978-1-61728-289-8

[11] Lemkin M A, Boser B E, Auslander D and Smith J H 1997 A 3-axis force balanced accelerometer using a single proof-mass Transducers '97: Int. Conf. on Solid State Sensors and Actuators vol 2 pp 1185-8

[12] Denoual M, Delaunay S, Allègre G and Robbes D 2009 Capacitively coupled electrical substitution for resistive bolometer enhancement Meas. Sci. Technol. 20015105

[13] Galeazzi M and McCammon D 2003 Microcalorimeter and bolometer model J. Appl. Phys. 93 4856-69

[14] Freire R C S, Catunda S Y C and Luciano B A 2009 Applications of thermoresistive sensors using the electric equivalence principle IEEE Trans. Instrum. Meas. 58 1823-30

[15] Allègre G, Guillet B, Robbes D, Méchin L, Lebargy S and Nicoletti $\mathrm{S} 2007$ A room temperature $\mathrm{Si}_{3} \mathrm{~N}_{4} / \mathrm{SiO}_{2}$ membrane-type electrical substitution radiometer using thin film platinum thermometers Meas. Sci. Technol. 18 183-9 
[16] Denoual M, Lebargy S and Allègre G 2010 Digital implementation of the capacitively coupled electrical substitution for resistive bolometers Meas. Sci. Technol. 21015205

[17] Oliveira A, Palma L S, Costa A S, Freire R C S and de C Lima A C 2006 A constant temperature operation thermoresistive sigma-delta solar radiometer Measurement 39 267-73

[18] Denoual M, Brouard D, Veith A, Pouliquen M, de Sagazan O, Attia $P$ and Allègre $G 2013$ Design of a sigma-delta interface for heat balanced bolometer Sensors Transducers $1833-46$ 